\title{
Effect of contrast and gaps between Vernier stimulus elements on sweep visual evoked potential measurements of human cortical Vernier responses
}

\author{
Russell D. Hamer ${ }^{1,2}$, Fabio Alves Carvalho ${ }^{1}$, and Dora Fix Ventura ${ }^{1}$ \\ 1- Universidade de São Paulo, São Paulo, SP, Brazil \\ 2- Smith-Kettlewell Eye Research Institute, San Francisco, CA, United States
}

\begin{abstract}
The present paper focuses on a classic hyperacuity, Vernier acuity — the ability to discriminate breaks in the collinearity of lines or edges on the order of only arcseconds of visual angle. We measured steady-state sweep visual evoked potentials (sVEPs) in response to $6 \mathrm{~Hz}$ periodic breaks in collinearity (Vernier offsets) in horizontal squarewave gratings. Vernier thresholds, estimated by extrapolating the amplitude of the first harmonic $(1 \mathrm{~F})$ to $0 \mu \mathrm{V}$, were measured for gratings with $4 \%, 8 \%$, $16 \%$, $32 \%, 64 \%$, and $80 \%$ contrast, with gaps of 0,2 , or 5 arcmin introduced between neighboring bar elements that formed the Vernier offsets. Thresholds for the $2 \mathrm{~F}$ response component provided an estimate of motion thresholds. The data confirmed and extended evidence that the odd- and even-harmonic components reflect cortical activity of different neurons (i.e., neurons that respond asymmetrically to the periodic breaks in alignment and neurons that respond symmetrically to the local relative motion cue of the stimulus). Suprathreshold data (peak amplitude, response slope, and response phase at the peak amplitude) provided additional independent evidence of this notion. Vernier thresholds decreased linearly as contrast increased, with a slope of approximately -0.5 on log-log axes, similar to prior psychophysical results. The form of contrast dependence showed more similarity to measures of magnocellular ganglion cell spatial precision than measures from parvocellular ganglion cells. Our data thus support the hypothesis that magnocellular ganglion cell output from the retina has the requisite properties to support cortical calculation of Vernier offsets at a hyperacuity level. Keywords: Vernier acuity, effect of contrast and gap, sweep VEP, motion responses, magnocellular signals.
\end{abstract}

Received 25 June 2012; received in revised form 23 November 2012; accepted 14 December 2012. Available online 18 November 2013.

\section{Introduction}

Humans and nonhuman primates are extraordinarily sensitive to breaks in the collinearity of lines and edges. Adults with normal vision can detect breaks in collinearity on the order of arcseconds of visual angle, as much as 10- to 30-times more precise than the dimensions of the stroke elements of standard 20/20 eye chart letters ( 1 arcmin), yielding the reliable detection of line/edge offsets that are substantially smaller than the dimensions of the foveal cone lattice. Reliable detection

Russell D. Hamer, Laboratório de Psicologia Sensorial, Departamento de Psicologia Experimental, Instituto de Psicologia, Universidade de São Paulo, São Paulo, Brasil, and Affiliate Scientist, Smith-Kettlewell Eye Research Institute, San Francisco, CA, USA. Fabio Alves Carvalho and Dora Fix Ventura, Laboratório de Psicologia Sensorial, Departamento de Psicologia Experimental, Instituto de Psicologia, Universidade de São Paulo, São Paulo, Brasil. Correspondence regarding this article should be directed to: Russell D. Hamer, Laboratório de Psicologia Sensorial, Departamento de Psicologia Experimental, Instituto de Psicologia, Universidade de São Paulo, Cidade Universitária, São Paulo, SP, 05508-030, Brasil. E-mail: russhamer2@, gmail.com of offsets as small as $2 \operatorname{arcsec}(0.033$ arcmin) have been measured (Westheimer \& McKee, 1977).

This ability was recognized centuries ago and was the basis for improvements in an ancient navigation tool, the astrolabe, introduced by Pedro Nunes in 1542 (cited in Medeiros, Farias de Medeiros \& Monteiro, 2004). Almost a century later in 1631, Pierre Vernier invented the Vernier caliper, which permitted unprecedented accuracy of measurement of the dimensions of objects by taking advantage of the exquisite sensitivity of the human visual system to misalignments of lines and edges. The systematic study of Vernier sensitivity appears to have begun with Wulfing (1892) and Hering (1899; cited in Westheimer \& McKee, 1977).

Since that time, Vernier acuity has been psychophysically investigated in many studies (e.g., Westheimer \& Hauske, 1975; Westheimer \& McKee, 1977; Levi \& Klein, 1982a,b, 1985; Morgan, Watt \& McKee, 1983; Morgan, 1986; Morgan \& Aiba, 1985a,b; Wilson, 1986, 1991; Bradley \& Skottun, 1987; McKee \& Levi, 1987; Wehrhahn \& Westheimer, 1990; Hu, Klein \& Carney, 1993; Levi, Klein \& Wang, 1994a,b; Carney \& Klein, 1997; Waugh \& Levi, 1993a,b,c; Norcia, Wesemann \& Manny, 1999; Levi, Klein \& Carney, 2000; Hou, Good \& Norcia, 2007). Additionally, cortical 
responses to Vernier offsets have been measured using visual evoked potentials (VEPs; Zemon \& Ratliff, 1982, 1984; Levi, Manny, Klein \& Steinman, 1983; Steinman, Levi, Klein \& Manny, 1985; Zak \& Berkley, 1986; Victor \& Conte, 2000; Norcia, Wesemann \& Manny, 1999; Skoczenski \& Norcia, 1999, 2002; Wesemann, Norcia \& Manny, 1996; Manny, 1988; Skoczenski \& Good, 2004; Mirabella, Kjaer, Norcia, Good \& Madan, 2006; Good \& Hou, 2004; Chen, Norcia, Pettet \& Chandna, 2005; Hou et al., 2007; Hou, Norcia, Madan, Tith, Agarwal \& Good, 2011).

\section{Visual evoked potentials are extraordinarily sensitive to breaks in collinearity}

Levi et al. (1983) showed that transient breaks in the collinearity of horizontal lines elicited a large transient VEP whose amplitude $(\mu \mathrm{V})$ scaled systematically and linearly with the log of Vernier-displacement magnitude (in $\operatorname{arcsec}$ ). Adopting the method used by Campbell \& Maffei (1970) to obtain VEP estimates of contrast thresholds, Levi et al. (1983) fitted a line to the peak amplitude vs. log-Vernier-offset data and extrapolated to $0 \mu \mathrm{V}$. The Vernier (VRN) thresholds obtained in this manner were on the order of 7 to 11 arcsec, close to the psychophysical threshold measured under the same conditions. This seminal work of Levi et al. (1983) was then extended and confirmed by Steinman et al. (1985) and Zak \& Berkley (1986). Zak \& Berkley (1986) were able to record significant VEP responses to breaks in the collinearity of a single line.

Time-domain analyses of transient VEPs have an intrinsic difficulty in that a Vernier offset cue and motion cue are presented simultaneously, and VEP responses specific to these cues overlap considerably in the time domain. The problem is how to distinguish the electrophysiological responses to one cue from the responses to the other cue. The use of steady-state VEPs and analysis of responses in the frequency domain more readily permit the disentanglement of these responses.

\section{Advantages of Vernier acuity measured with steady-state sweep visual evoked potential}

The existence of response asymmetry found in early transient VEP studies (i.e., a stronger response to misalignment and weaker response to realignment) implies that the frequency spectrum of the steady-state VEP in response to this kind of stimulus will contain both odd and even harmonics of the stimulus temporal frequency, thus permitting the measurement of Vernierspecific response components. Zemon \& Ratliff (1982, 1984) and Zemon, Victor \& Ratliff (1986) were the first to show this using the frequency domain analysis of steady-state VEPs.

In transient VEPs, the response contains many temporal frequencies, and extraction of the evoked response may require averaging the response across many trials for each displacement magnitude (Regan, 1989). In contrast, one advantage of steady-state VEPs is that one knows in advance at which temporal frequencies a stimulus-driven response is expected to occur. Frequency-domain analysis of steady-state evoked responses focuses on a very narrow band of the full electroencephalographic spectrum and thus can yield a much higher signal-to-noise ratio (SNR) for substantially shorter recording epochs.

Norcia et al. (1999) took advantage of the early observations of asymmetry in transient VEP responses to VRN stimuli and combined the frequency-domain approach of Zemon \& Ratliff $(1982,1984)$ with the efficient steady-state sweep VEP (sVEP) paradigm (Regan, 1973; Tyler, Apkarian, Levi \& Nakayama, 1979; Norcia \& Tyler, 1985) to measure VRN thresholds.

The present study extends prior steady-state VEP measures of VRN responses by exploring the effect of stimulus contrast on both VRN thresholds and suprathreshold response features (i.e., amplitude, phase, and slope). Prior sVEP studies of VRN examined responses to high-contrast stimuli (Norcia et al., 1999; Skoczenski \& Norcia, 2002; Chen et al., 2005; Hou et al., 2007). We evaluated our results in relation to the hypothesis that magnocellular but not parvocellular retinal output has the requisite properties to support extraction of a psychophysically accessible signal in the visual cortex (Lee, Wehrhahn, Westheimer \& Kremers, 1993, 1995; Wachtler, Wehrhahn \& Lee, 1996; Rüttiger \& Lee, 2000; Rüttiger, Lee \& Sun, 2002; Sun, Lee \& Rüttiger, 2003; Sun, Rüttiger \& Lee, 2004; Lee, Rüttiger $\&$ Sun, 2005; Sun et al., 2008).

\section{Methods}

Sweep VEP measures of Vernier (VRN) offsets have been described elsewhere (Norcia et al., 1999; Skoczenski \& Norcia, 1999, 2002; Skoczenski \& Good, 2004; Chen et al., 2005; Hou et al., 2007; Hou et al., 2011). The methods are briefly summarized here.

PowerDIVA software and hardware (e.g., Chen et al., 2005; Hou et al., 2007) were used to generate the stimuli, record and digitize the incoming electroencephalogram, and perform the online frequency analyses.

\section{Stimuli}

Vernier (VRN) protocol (Figure 1A). Vernier offsets were introduced to $2 \mathrm{c} / \mathrm{deg}$ horizontal squarewave gratings presented on a 21-inch FIMI Model MD0709BRM-MGD 403 monitor $(60 \mathrm{~Hz}$, 1600 horizontal $\times 1200$ vertical resolution) at a mean luminance of $161 \mathrm{~cd} / \mathrm{m}^{2}$. The grating dimensions were $8.8 \mathrm{deg}$ high $\times 9.2 \mathrm{deg}$ wide and viewed from $175 \mathrm{~cm}$. For some conditions (e.g., high contrast and zero gap), the measurement of Vernier thresholds from this distance demands the presentation of subpixel offsets (for our monitor, viewed at $175 \mathrm{~cm}$, each pixel subtended 0.479 min, larger than the offsets used at the beginning of our sweeps). The PowerDIVA system was able to generate subpixel offsets that were achieved using a centroiding algorithm (Alexander \& Ng, 1991; Krotkov, 1986).

The Vernier stimulus protocol is illustrated in Figure 1A. To generate the Vernier offsets, the gratings 
were broken into vertical columns of bar elements, half of which were stationary (columns 1,3 , and 5 in Figure 1A) and half of which alternated at $6 \mathrm{~Hz}$ between two states (columns 2, 4, and 6)-aligned (AL) and misaligned (MAL) - with the stationary bars. Each bar element subtended 45 arcmin horizontally.

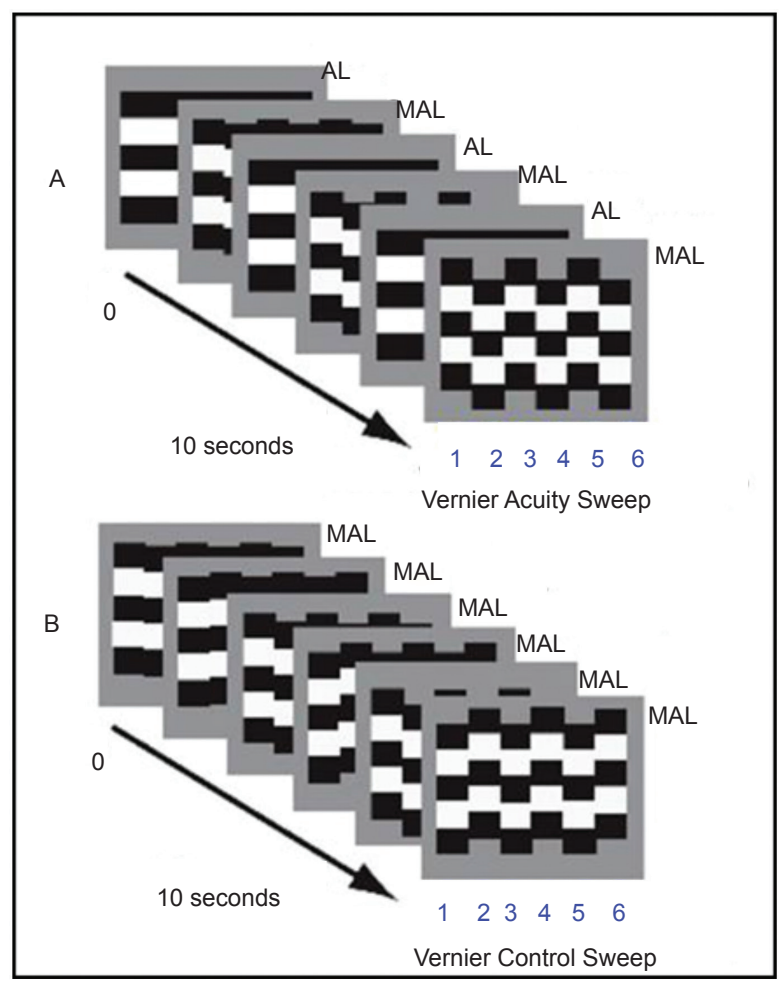

Figure 1. For each protocol the sequence of the panels along the arrow shows the states of the stimulus pattern presented over time. For didactic purposes, only six columns of bars are shown. The actual experiment had a total of 12 columns of bars, so a total of 418 Vernier offsets were presented across the entire stimulus array. One column of bars changes position (columns 2,4 , and 6) in each half-cycle of the temporal modulation of the stimulus, and one set remains stationary (columns 1, 3, and 5). The moving bars alternate at the chosen temporal frequency (TF; $6 \mathrm{~Hz}$ in this case) between the two states. For the VRN protocol (A), the two states are: bars aligned (AL) across the stimulus screen and bars misaligned (MAL). The misalignment always occurred in one direction. In panel A, the misalignment is downward (bars in columns 2, 4, and 6 shifted down during the displacement). For the motion control protocol (B), the two states are: displaced bars misaligned (MAL) downward, below the static bars, across the stimulus screen, and bars misaligned (MAL) above the static bars.

Motion control protocol (Figure 1B). In this protocol, the identical motion was generated by displacements of the moving columns of bars (columns 2, 4, and 6 in Figure 1B), but the motion was symmetric. After moving downward, the bars did not return to collinearity but instead were displaced an equal distance above the neighboring stationary bars (columns 1, 3, and $5)$. The only difference between this stimulus sequence and the stimulus in the VRN protocol (Figure 1A) was that the control stimulus never presented an aligned pattern and all of the stimulus components were thus symmetric.

Gap experiment. In the main contrast experiment, the grating elements that underwent the Vernier displacements (i.e., misalignments) were abutting (i.e., zero gap). We also ran the VRN protocol while introducing gaps between the elements. Two non-zero gaps were tested-2' and 5'-based on prior data that showed that the majority of the effects of the gaps on VEP amplitude and threshold in Vernier tasks occurred within a few arcmin of the gap (Zemon \& Ratliff, 1982; Levi et al., 1983; Steinman et al., 1985; Norcia et al., 1999; Hou et al., 2007).

During each sVEP trial, the amplitude of the offset was increased logarithmically over 101 -s recording time bins, starting from near or below the threshold to well above threshold. The sweep range was adjusted to be appropriate for the contrast or gap condition used. The two sweep ranges used were $0.15-5$ arcmin for the three highest contrasts and $0.25-7.5$ arcmin (both about 5 octaves). The maximum offset permitted was 7.5 arcmin because we used a $2 \mathrm{c} / \mathrm{deg}$ stimulus and chose to restrict the displacements to $\leq 90$-deg spatial phase.

\section{Sweep visual evoked potential data analysis}

The incoming electroencephalogram was filtered by the recording amplifier (Grass Model 12) between 0.3 and $100 \mathrm{~Hz}$ with a gain of 50,000. The electroencephalogram was digitized at $607 \mathrm{~Hz}$. The resulting steady-state VEP was analyzed at the stimulus frequency $(1 \mathrm{~F}=6 \mathrm{~Hz})$ and $2^{\text {nd }}$ harmonic $(2 \mathrm{~F}=12 \mathrm{~Hz})$. The amplitude and phase at these harmonics were extracted. Thresholds for the $1 \mathrm{~F}$ and $2 \mathrm{~F}$ responses were derived by extrapolating along a line fit to the rising phase of the SVEP response to 0 $\mu \mathrm{V}$ (Campbell \& Maffei, 1970; Norcia \& Tyler, 1985; Norcia, Tyler, Hamer \& Wesemann, 1989). The sVEP response that was used to estimate the threshold for the $1 \mathrm{~F}$ and $2 \mathrm{~F}$ components was the vector average of 10 trials for each contrast/gap condition tested.

The sVEP signals at $1 \mathrm{~F}$ or $2 \mathrm{~F}$ that were used to perform the extrapolation to threshold were required to adhere to strict amplitude, phase, and cross-trial-coherence (Tcirc ${ }^{2}$; Victor \& Mast, 1991) criteria to ensure that the responses in the bins used were significantly above noise (Chen et al., 2005). Local noise for each recording bin was estimated by deriving the amplitude and phase of the sVEP $1 \mathrm{~Hz}$ above and below each of the analysis harmonics. The vector average of the responses at these two frequencies constituted the local noise estimate for each analysis bin. The SNR was enhanced by applying adaptive filtering of the data using a recursive leastsquares algorithm (Tang \& Norcia, 1993, 1995).

\section{Procedures: main contrast experiment}

Fourteen young adults (20-33 years old) participated in the contrast experiment: seven males (mean age, 28.6 \pm 3.3 years) and seven females (mean age, $27.9 \pm 2.5$ years). All participants had 20/20 corrected Snellen acuity or better and were wearing their correction (if needed). 

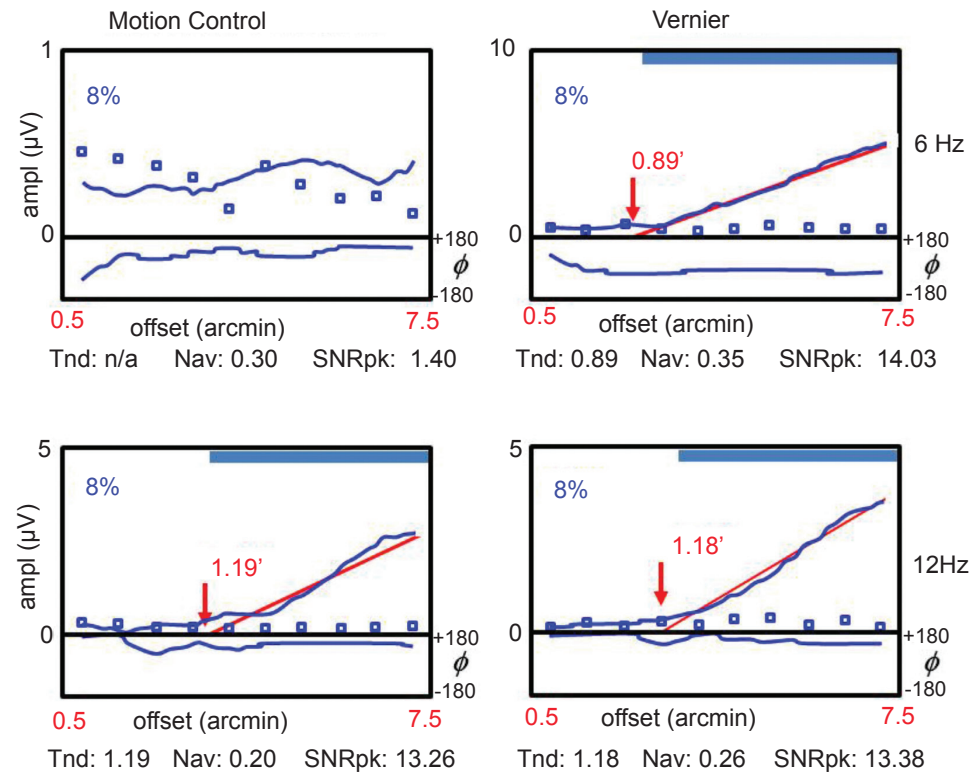

Figure 2. Sweep VEP responses from one subject tested with a low contrast grating (8\%). The panels on the left and right show the results for the motion control and VRN protocols, respectively. In each of the four panels, the upper and lower subpanels show the response amplitude $(\mu \mathrm{V})$ and response phase $(-180$ to $+180 \mathrm{deg})$, respectively. Stimulus offsets were swept logarithmically from $0.5^{\prime}$ to $7.5^{\prime}$. The solid blue curves in each panel are the Fourier-analyzed response amplitudes at $6 \mathrm{~Hz}$ (i.e., $1 \mathrm{~F}$, top two panels) and $12 \mathrm{~Hz}$ (i.e., 2F, bottom two panels). The small blue squares show the estimate of the local noise in each 1-s analysis epoch, and their average is given by the value Nav. SNRpk is the peak signal-to-noise ratio achieved. Thresholds (red arrows) are derived from the intersection of the red extrapolation lines with $0 \mu \mathrm{V}$ on the amplitude axis. The Vernier threshold (1F) was 0.89 '. The threshold for the $2^{\text {nd }}$ harmonic in the VRN protocol was 1.18 ', which was almost identical to the threshold for the motion control (1.19'). Notice that there was no significant signal at $1 \mathrm{~F}$ in the motion control protocol. The horizontal blue bars at the top of each panel mark the bins in which the signal was statistically significant according to the Tcirc ${ }^{2}$ statistic (Victor \& Mast, 1991).

During each sVEP trial, the participant looked binocularly at the center of the stimulus while the steady-state VEP was recorded from three unipolar electrodes placed on the scalp $(\mathrm{O} 1, \mathrm{Oz}$, and $\mathrm{O} 2 ; 10 / 20$ system; Odom et al., 2004), with a reference at $\mathrm{Cz}$ and ground at Fz. No fixation stimulus was used in order to minimize the possibility of generating a spurious Vernier or relative motion signal.

Data used to represent the response for a given condition were those from the channel with the lowest threshold, assuming that all of the amplitude, phase, and Tcirc $^{2}$ criteria were satisfied (Norcia \& Tyler, 1985; Norcia et al., 1989).

sVEP responses were measured for the VRN and motion control protocols for gratings presented at six different contrasts in a single recording session: $4 \%, 8 \%, 16 \%, 32 \%, 64 \%$, and $80 \%$. Three of the 14 participants failed to generate reliable sVEP thresholds at $4 \%$ contrast, and one participant was not tested at $80 \%$ contrast. The test sequence within each session proceeded from low to high contrast to minimize any effects of contrast adaptation (Nelson, Seiple, Kupersmith \& Carr, 1984; Norcia et al., 1989).

\section{Procedures: gap experiment}

Eight of the 14 participants also participated in the gap experiment: four males (mean age, $29.3 \pm 4.1$ years) and four females (mean age, $27 \pm 2.4$ years). For each of the gap conditions $\left(0,2^{\prime}\right.$, and 5 '), four contrasts were tested: $4 \%, 16 \%, 32 \%$, and $64 \%$. The remaining procedures were the same as those used in the main contrast experiment. This research was conducted in accordance with the Helsinki Declaration.

\section{Results}

Example of individual sVEP responses from one normal adult tested at two grating contrasts $(8 \%$ and $32 \%$ ) are shown in Figures 2 and 3.

\section{Association of $1 F$ and $2 F$ response components with Vernier and motion stimulus components in the VRN protocol}

Prior studies that applied frequency-domain analyses of steady-state VEPs provided several lines of evidence that the $1 \mathrm{~F}$ (odd harmonic) response component derives from cortical cells that are able to discriminate between the two states of the stimulus (i.e., the Vernier offset [the misalignment event] and the return to alignment). Because these two stimulus phases generate different response amplitudes (Zemon \& Ratliff, 1982; Levi et al., 1983), they generate strong oddharmonic response components, especially at $1 \mathrm{~F}$. The pure motion and contrast components of the stimulus in the VRN protocol were both symmetric and thus elicited responses only at the even harmonics $(2 \mathrm{~F}, 4 \mathrm{~F}$, etc.).

Motion control protocol. A simple control experiment (Figure 1, second panel) confirmed that the odd-harmonic ( $1 \mathrm{~F}$ in our analyses) components indeed reflected responses that were specific to the Vernier 

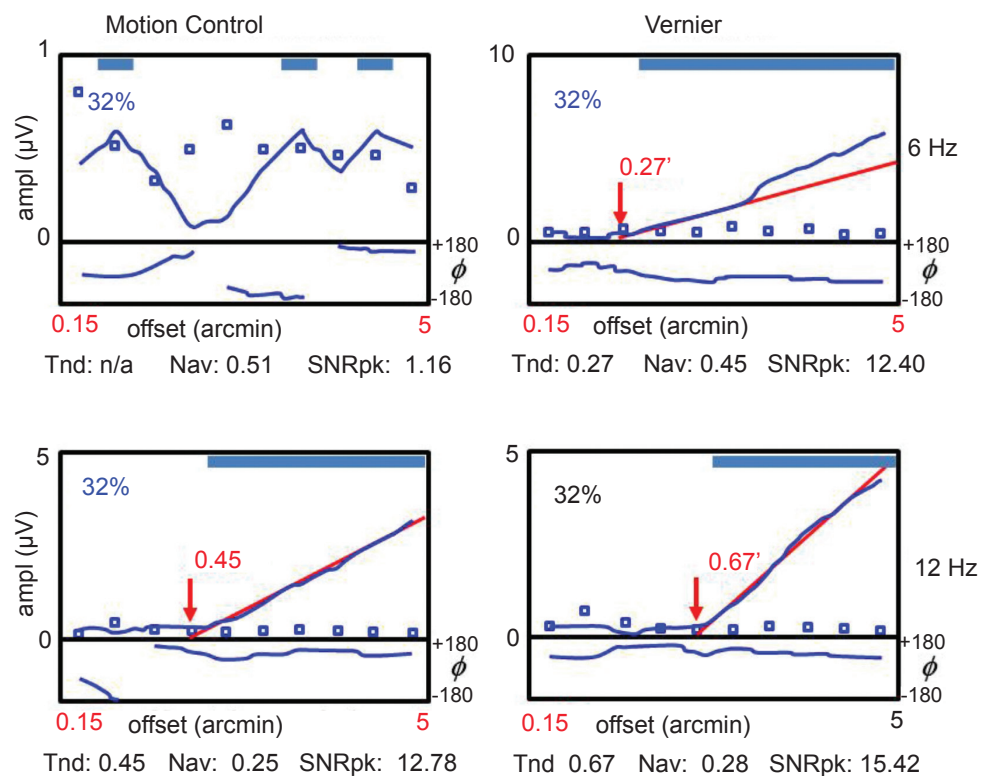

Figure 3. Sweep VEP responses from one subject tested with gratings at $32 \%$ contrast. These data are in the same format as in Figure 2. The same subject was tested using a grating at $32 \%$ contrast. In this case, the stimulus offset was swept from .15' to 5'. Note that the Vernier (1F) threshold is lower at this higher contrast (.27' or 16 arcsec).

breaks in alignment. In adults with normal vision, we generally see only even-harmonic responses to the motion control stimulus (Norcia et al., 1999; Chen et al., 2005; Hou et al., 2007). ${ }^{1}$

Examples from one subject tested with the motion control protocol at two contrasts $(8 \%, 32 \%)$ are shown in the left panels in Figures 2 and 3. Notice that there was no significant $1 \mathrm{~F}$ response at either contrast tested. However, there was a large response at both $1 \mathrm{~F}$ and $2 \mathrm{~F}$ when the subject was tested with the VRN protocol (right panels). These results were typical of the data from all subjects.

\section{Effects on sweep visual evoked potential thresholds of contrast and gaps between stimulus elements}

Contrast-dependence of Vernier and motion thresholds. Figure 4 shows the geometric mean of the $1 \mathrm{~F}$ and $2 \mathrm{~F}$ thresholds $( \pm 1 \mathrm{SE}) v s$. log grating contrast. Thresholds from the 1F component (solid black curve and circles) represent the asymmetrical cortical response to the Vernier breaks in the gratings (i.e., the alternation between AL and MAL states in the VRN stimulus).

1. When tested monocularly, the symmetrical horizontal motion of vertical sinewave gratings elicits strong oddharmonic responses in normal young infants and toddlers with esotropia and adults with a history of esotropia (Norcia, Garcia, Humphry, Holmes, Hamer \& Orel-Bixler, 1991; Jampolsky, Norcia \& Hamer, 1994; Norcia, Hamer, Jampolsky, Orel-Bixler, 1995; Birch, Fawcett \& Stager, 2000). Motion asymmetries can occasionally occur in normal adults, but when they do, they are less pronounced and less prevalent for vertical motion than for horizontal motion (Shea, Chandna \& Norcia,1999; Chen et al., 2005). One can thus minimize the likelihood or magnitude of intrusion of odd-harmonic motion responses by testing using horizontal gratings.
Figure 4 also compares the sVEP VRN threshold data with typical psychophysical data (Sun et al., 2003). The contrast-dependence of the $1 \mathrm{~F}$ cortical thresholds was nearly identical to the contrast-dependence of human psychophysical Vernier thresholds vs. the contrasts obtained in Sun et al. (solid red). Both the Sun et al. (2003) psychophysical data and our 1F VEP data are fit well by a line with slope $=-0.5$ on log-log axes (solid black line), similar to slopes measured in some prior psychophysical studies (e.g., Watt \& Morgan, 1983; Krauskopf \& Farrell, 1991; Wehrhahn \& Westheimer, 1990; Sun et al., 2004).

The thresholds from the $2 \mathrm{~F}$ component (solid blue curve and circles) had strikingly different contrast dependence with almost no changes in threshold until after $16 \%$ contrast. $^{2}$ The $2 \mathrm{~F}$ thresholds are interpreted as responses to the local motion of the moving grating elements, which is inherently symmetric. This interpretation is supported by the similarity of the $2 \mathrm{~F}$ data from the VRN stimulus and the $2 \mathrm{~F}$ data from the symmetrical motion control stimulus (dashed blue), which elicits only $2 \mathrm{~F}$ and no $1 \mathrm{~F}$.

Effect of gaps between stimulus elements on Vernier and motion thresholds. The early transient VEP study of Steinman et al. (1985) showed that Vernier responses were reduced by introducing separations (gaps) between the Vernier stimulus elements. This

2. The $1 \mathrm{~F}$ thresholds (solid black curve and data in Figure 4) have significantly different contrast dependence than the $2 \mathrm{~F}$ thresholds (solid blue), reflected by a significant harmoniccontrast interaction $\left(F_{1,143}=12.32, p<.001\right.$, mixed linear model with a compound symmetry variance-covariance structure $)$ and significant main effects of harmonic $\left(F_{1,143}=\right.$ $19.02, p<.0001)$ and contrast $\left(F_{1,143}=82.79, p<.0001\right)$. 


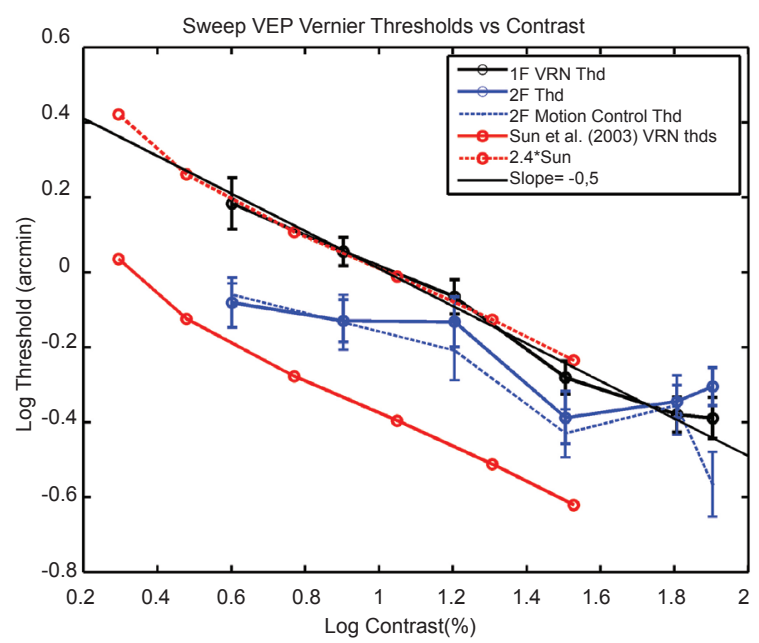

Figure 4. Sweep VEP VRN (1F) and motion (2F) thresholds as a function of grating contrast compared with psychophysical VRN thresholds from Sun et al. (2003). Data from the VRN protocol (solid black curve, 1F; blue curve, 2F) are the means of the log sVEP thresholds \pm 1 SE from 14 subjects, with the exception of the data at $4 \%(n=11)$ and $80 \%(n=13)$. Motion control (2F) data (dashed blue curve) are the means of the $\log \mathrm{sVEP} \pm 1 \mathrm{SE}$ from $11,6,12,12,12$, and 6 subjects for the $4 \%, 8 \%, 16 \%, 32 \%, 64 \%$, and $80 \%$ contrast conditions, respectively. Thresholds from the $1 \mathrm{~F}$ component represent the asymmetric cortical response to the alternation between $\mathrm{AL}$ and MAL states in the VRN stimulus. Note that the contrastdependence of the $1 \mathrm{~F}$ cortical thresholds are nearly identical to the contrast-dependence of human psychophysical Vernier thresholds $v s$ contrast obtained in Sun et al. (2003; solid red). Data of Sun et al. were shifted up by a factor of 2.4 (dashed red) to highlight the similarity of to the slope of the sVEP data. Both the psychophysical data and 1F VEP data are fit well by a line with slope $=-.5$ on log-log axes (sold black line). Our $1 \mathrm{~F}$ thresholds at high contrast are similar to prior sVEP measures. For example, the average $1 \mathrm{~F}$ threshold at $80 \%$ contrast (with no gap) was .63 arcmin in Figure 5 in Hou et al. (2007). The thresholds from the $2 \mathrm{~F}$ component (solid blue curve and circles) have a strikingly different contrast dependence with almost no change in threshold until after $16 \%$ contrast. The $2 \mathrm{~F}$ thresholds are interpreted as responses to the local motion of the moving grating elements, which is inherently symmetric. This interpretation is supported by the similarity of the $2 \mathrm{~F}$ data from the VRN stimulus to the $2 \mathrm{~F}$ data from the symmetrical motion control stimulus (dashed blue), which elicits only $2 \mathrm{~F}$ and no $1 \mathrm{~F}$.

finding was extended to steady-state measures of Vernier responses using high-contrast $(80 \%)$ Vernier stimuli (Norcia et al., 1999; Hou et al., 2007).

Prior work showed that gaps affect both sVEP amplitudes and the extrapolated thresholds (Norcia et al., 1999; Hou et al., 2007). We first examined the effect on thresholds. Figure 5 shows the mean thresholds for the $1 \mathrm{~F}$ (left) and $2 \mathrm{~F}$ (right) response components from the VRN protocol. The results for four contrasts are shown for each of the gaps.

Notice that the introduction of the gaps increased $1 \mathrm{~F}$ thresholds but had no effect on $2 \mathrm{~F}$ thresholds for gratings with the three highest contrasts $(16 \%, 32 \%$, and
$64 \%) .{ }^{3}$ The contrast-independence of the $2 \mathrm{~F}$ thresholds is consistent with the notion that the mechanism that generates the $2 \mathrm{~F}$ component is not comparing neuronal activity across the spatial boundaries between the stimulus elements. The increase in $2 \mathrm{~F}$ thresholds at $4 \%$ contrast was unexpected and will be addressed in the Discussion section below.

The neurons that underlie the $1 \mathrm{~F}$ response components have lateral interactions; thus, the $1 \mathrm{~F}$ thresholds are affected by gaps, causing thresholds to elevate for small gaps on the order of resolution acuity. This increase in thresholds in the sVEP for $1 \mathrm{~F}$ is similar to the effect of gaps in psychophysical tests of Vernier threshold (e.g., Norcia et al., 1999; Hou et al., 2007).

\section{Contrast dependence of suprathreshold response features}

Suprathreshold VEP response features, which are not accessible in psychophysical threshold measures, can provide additional important insights into the underlying neural mechanisms. The response features examined in the present study were the following: (i) peak response amplitude as a function of grating contrast, (ii) sVEP slope as a function of contrast, (iii) sVEP minimum response phase as a function of contrast, and (iv) effect of gaps (separation between VRN stimulus elements) on peak amplitude. In all four cases presented below, we found that the $1 \mathrm{~F}$ and $2 \mathrm{~F}$ components manifested different contrast dependence.

Contrast dependence of peak response amplitude. The dependence of sVEP peak amplitude on grating contrast is shown in Figure 6. For each grating contrast, we recorded the peak amplitude of both the $1 \mathrm{~F}$ $\left(\mathrm{A}_{\mathrm{pklF}}\right)$ and $2 \mathrm{~F}\left(\mathrm{~A}_{\mathrm{pk} 2 \mathrm{~F}}\right)$ components. This was repeated for each subject, and the average $\mathrm{A}_{\mathrm{pklF}}$ and $\mathrm{A}_{\mathrm{pk} 2 \mathrm{~F}} v s$ contrast is shown in Figure 6.

Note that the peak 1F (solid black) and 2F (solid blue) amplitudes both increased with contrast and exhibited a plateau at contrasts $>16 \%$. However, they had quite distinct contrast dependence; the $1 \mathrm{~F}$ amplitudes were larger and grew much more rapidly up to the plateau. The 2F peak amplitudes derived from the motion control protocol are shown as dashed blue lines. They closely paralleled the $2 \mathrm{~F}$ peak amplitudes from the VRN protocol but were slightly lower at all but the lowest contrast. Hou et al. (2007) tested at high contrast $(80 \%)$ and also found that the $2 \mathrm{~F}$ amplitudes from the motion control protocol tended to be lower than the 2F amplitudes from the VRN protocol despite the fact that local contrast and motion cues were the same in both. They suggested that the $2^{\text {nd }}$ harmonic response to the motion can encode, to some degree, the spatial

3. For the 1F data (left panel), we found main effects of gap $\left(F_{1,74}=6.93, p=.01\right)$ and contrast $\left(F_{1,74}=14.54, p<.001\right)$ but no significant gap $\times$ contrast interaction $\left(F_{1,74}=.44, p=\right.$ .51 , mixed linear model with a compound symmetry variancecovariance structure). For the $2 \mathrm{~F}$ data, no significant main effects or interactions were found. 

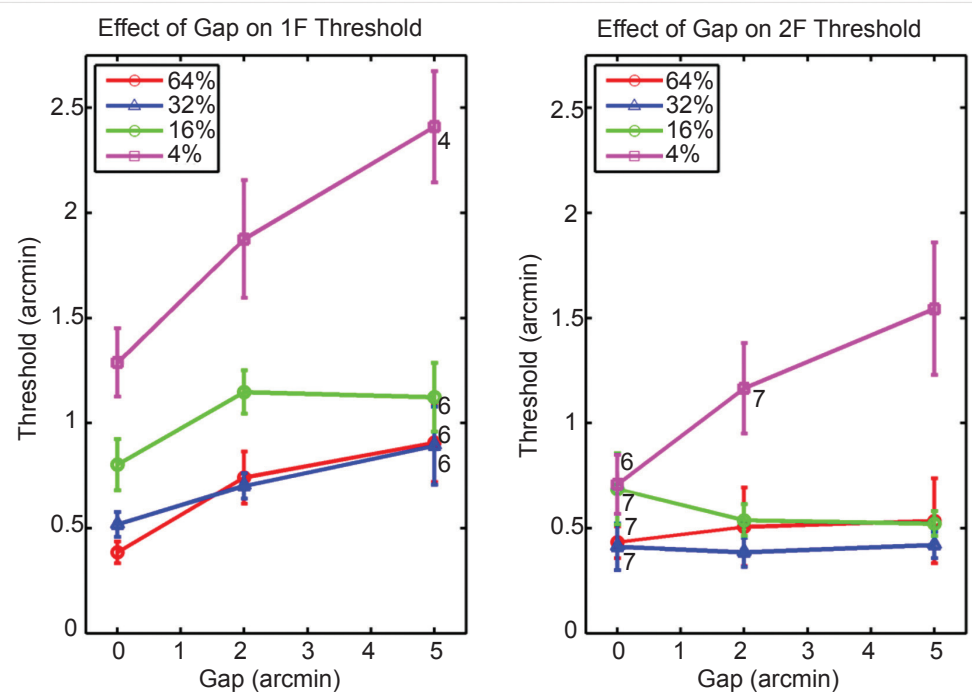

Figure 5. Gaps introduced between the stimulus elements increased thresholds for 1F (Vernier) but had little effect on $2 \mathrm{~F}$ thresholds at $16 \%, 32 \%$, and $64 \%$ contrasts. The $2 \mathrm{~F}$ thresholds measured at $4 \%$ contrast increased with gap size, although each of the $2 \mathrm{~F}$ thresholds were lower than comparable $1 \mathrm{~F}$ thresholds. Eight of the 14 subjects were tested in the gap protocols. The numbers next to some of the data points indicate the number of subjects for which reliable thresholds could be estimated whenever this number was $<8$.

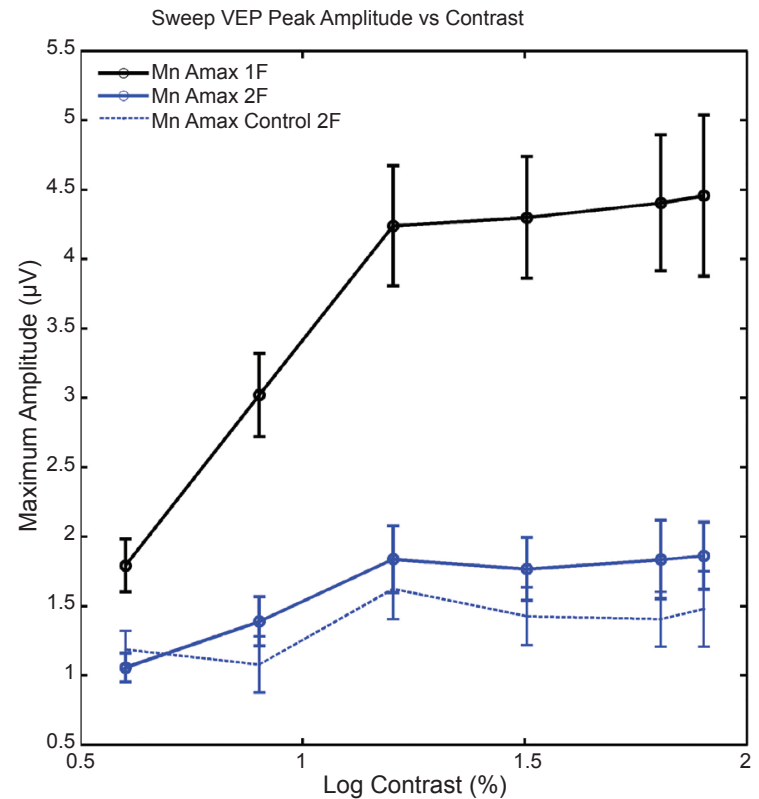

Figure 6. Peak amplitude for sVEP Vernier response components (1F; black) and motion responses (2F; blue) as a function of grating contrast. Note that the $2 \mathrm{~F}$ components from either the VRN protocol (solid blue) or motion control protocol (dotted blue) have much less dependence on contrast than the $1 \mathrm{~F}$ (Vernier) component for low-contrast stimuli. No single scaling factor can make the $1 \mathrm{~F}$ and $2 \mathrm{~F}$ data congruent. Note also that the $2 \mathrm{~F}$ peak amplitudes derived from the motion control protocol across contrast are lower than those from the Vernier protocol, consistent with prior research at high contrast (Hou et al., 2007).

configuration of the stimulus because this is the only aspect that differs between the two stimulus protocols. Our 2F data had the same behavior at high contrasts and showed the same tendency at all contrasts tested, with the exception of $4 \%$.

Contrast dependence of sVEP slope. The rate at which the sVEP response increases with displacement amplitude during the sweeps provides a gauge of the gain of the mechanism that generates the response component that is analyzed. Figure 7 shows the mean slopes derived from the extrapolation lines fit to each subject's sweep data at each contrast tested.

Two features are prominent in Figure 7. First, the response amplitude of the $1 \mathrm{~F}$ (Vernier) component clearly increases faster with displacement magnitude during sVEP trials than the $2 \mathrm{~F}$ components measured simultaneously: for each contrast condition tested, the mean $1 \mathrm{~F}$ slope was greater than the $2 \mathrm{~F}$ slope, ranging from 1.6- to 2.6-times greater. Second, the $2 \mathrm{~F}$ slopes show no dependence on contrast, whereas the $1 \mathrm{~F}$ slopes increase with contrast up to $\sim 16 \%$ and plateau at higher contrasts. The lack of contrast dependence for the $2 \mathrm{~F}$ slopes is also noteworthy because the extrapolated thresholds over the high-contrast range ( $\geq 16 \%$ ) decrease with contrast (Figure 4), implying that changing contrast in this range causes parallel shifts of the sVEP data along the vernier-displacement axis..

The slopes shown in Figure 7 can be transformed into Vernier displacement gain values in a form analogous to the contrast gain measured from singlecellular contrast response functions (e.g., Kaplan \& Shapley, 1982, 1986; Purpura, Kaplan \& Shapley, 1988). To measure Vernier displacement gain, we transformed the sVEP extrapolation lines that were used to estimate displacement thresholds into linear-linear coordinates. We then calculated the slope of the resulting analytic expression at a very small displacement value ${ }^{4}$ above the extrapolated threshold. This value was taken as the

4. We measured the slope between the extrapolated threshold value, where $\mathrm{SVEP}$ amplitude $=0$, and $.001 *$ incr, where incr $=$ the size of the increment in displacement (in log units) between any two of the 10 sweep bins. 


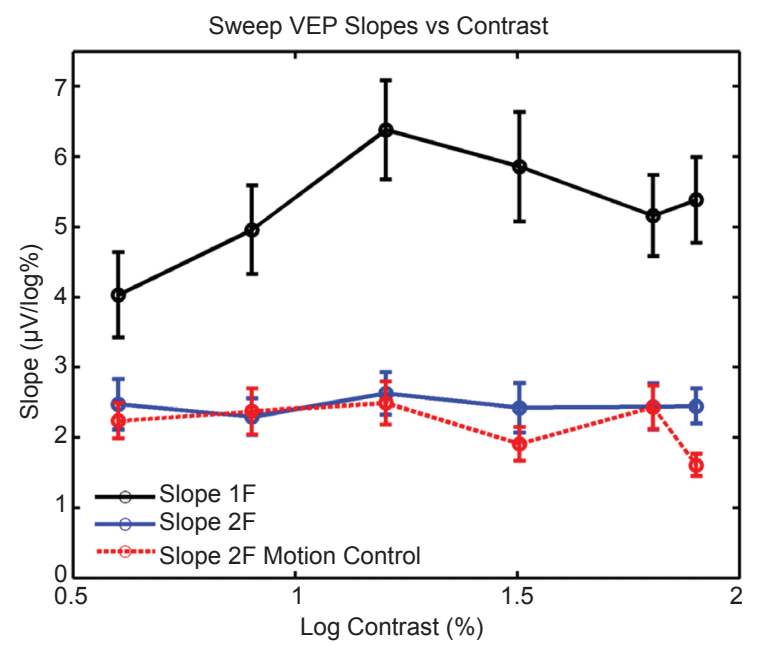

Figure 7. Mean slopes $( \pm \mathrm{SE})$ derived from the extrapolation lines fit to each subject's VRN and motion control sweep data at each contrast tested. Notice that (1) the slope for the $1 \mathrm{~F}$ component is always greater than the slope for the $2 \mathrm{~F}$ component (by a factor of 1.6- to 2.6), and (2) the slopes for the $1 \mathrm{~F}$ and $2 \mathrm{~F}$ components have different contrast dependence, with the $2 \mathrm{~F}$ data showing virtually no dependence on stimulus contrast.

estimated displacement gain. These gains are shown in Figure 8.

The gain functions for Vernier (1F) and motion (2F) have distinctly different contrast dependence. The Vernier-associated displacement gain increased linearly with $\log$ displacement between $4 \%$ and $80 \%$ contrast by a factor of 5.5. The $2 \mathrm{~F}$ gain function was lower than the $1 \mathrm{~F}$ function at all contrasts above $4 \%$ and was almost contrast-independent between $4 \%$ and $16 \%$ contrast. Between $16 \%$ and $32 \%$ contrast, the $2 \mathrm{~F}$ displacement gain increased by a factor of $\sim 2.2$ and saturated (and even decreased) at higher contrasts.

Contrast dependence of minimum response phase $\left(f_{\text {min }}\right)$. Phase generally decreases with the amplitude of the stimulus displacement, so the bin with the maximum SNR will generally have the lowest phase value (i.e., smallest phase lag). Thus, we term this the minimum phase $\left(f_{\min }\right)$ for that condition. $f_{\text {min }}$ can provide an estimate of the high-SNR response speed (minimum apparent latency) for each contrast condition.

For each subject's data and for each contrast tested, $\mathrm{f}_{\min }$ values were converted to apparent latencies (Hamer $\&$ Norcia, 1994). For $1 \mathrm{~F}(6 \mathrm{~Hz}), 360 \mathrm{deg}$ corresponds to $167.7 \mathrm{~ms}$, so 90 deg corresponds to an apparent latency ${ }^{5}$

5. The phase values used were the raw phases generated by the PowerDIVA software. The conversion to apparent latency generates a relative value and not an absolute cortical latency because latencies introduced by the equipment (e.g., analog filters, etc.) were not corrected for. However, changes in phase between contrast conditions or between displacement amplitudes within a given stimulus sweep can be converted to an absolute latency difference between the conditions (or sweep bins).

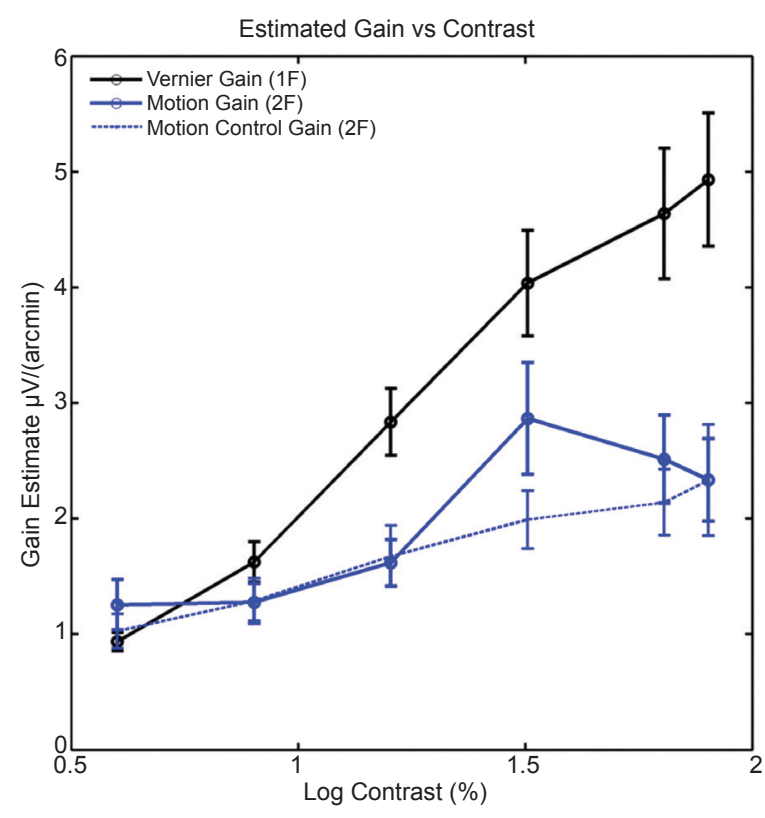

Figure 8. Estimated Vernier displacement gain as a function of grating contrast. For each contrast condition for each subject, the threshold extrapolation line was used as a proxy for the data. From the threshold and slope of the extrapolation line, we estimated the derivative at a small fixed increment above the threshold of the linearized sVEP data after conversion of the contrast values from $\log$ to linear units. This derivative constitutes an estimate of the displacement gain of the Vernier (1F; solid black) and motion (2F; blue) response components. Note that the Vernier and motion gains have different dependence on contrast. The Vernier gain increased linearly with log contrast over the full contrast range tested. The motion $(2 \mathrm{~F})$ gain was almost independent of contrast at low contrasts and then increased by a factor of $\sim 2$ starting at $16 \%$ contrast.

of $41.7 \mathrm{~ms}$, and $45 \mathrm{deg}$ corresponds to an apparent latency of $20.83 \mathrm{~ms}$, etc. For 2F (12 Hz), $360 \mathrm{deg}$ corresponds to $83.3 \mathrm{~ms}, 90 \mathrm{deg}$ corresponds to 20.83 $\mathrm{ms}$, and $45 \mathrm{deg}$ corresponds to $10.41 \mathrm{~ms}$, etc.

Figure 9 shows the mean apparent cortical latency for the $1 \mathrm{~F}$ and $2 \mathrm{~F}$ components for each contrast tested. The apparent latencies were then shifted to start at 0 $\mathrm{ms}$ for the $4 \%$ contrast condition. Absolute latency decreases relative to the latency at $4 \%$ contrast are thus readily seen. This defines the ordinate in Figure 9.

Figure 9 shows that cortical latencies to Vernier offsets (1F) decreased much more rapidly with increasing stimulus contrast than the motion-related responses (2F). This is especially apparent at the three lowest contrasts $(4 \%, 8 \%$ and $16 \%)$ for which the $2 \mathrm{~F}$ latencies were almost constant with contrast. Between $4 \%$ and $64 \%$ contrast, the apparent $1 \mathrm{~F}$ latency decreased by a total of $21 \mathrm{~ms}$, whereas the $2 \mathrm{~F}$ latency decreased by only $6.5 \mathrm{~ms}$. The different contrast dependence provides additional evidence that the $1 \mathrm{~F}$ and $2 \mathrm{~F}$ components derive from different cortical neural sources. 


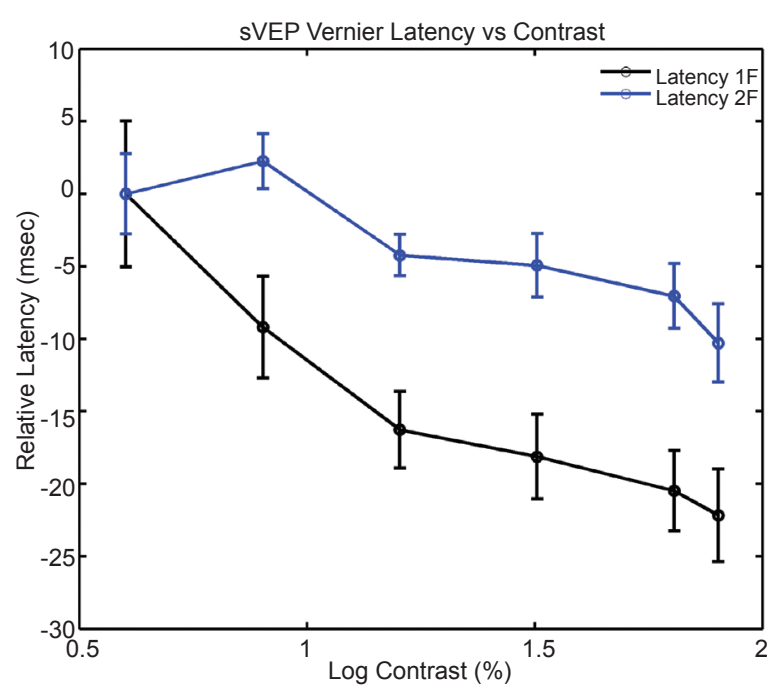

Figure 9. Mean $( \pm \mathrm{SE})$ relative apparent latencies as a function of grating contrast for $1 \mathrm{~F}$ (black) and $2 \mathrm{~F}$ (blue) components. For each subject's data and for each contrast tested, the phase (in deg) in the analysis bin with the highest SNR was extracted. Phase generally decreased with the amplitude of the stimulus displacement, so the bin with the maximum SNR generally had the lowest phase value $\left(f_{\min }\right)$. At each contrast, the $f_{\min }$ values were averaged across subjects. These mean $\mathrm{f}_{\min }$ values and their SDs and SEs were converted to apparent latency (Hamer \& Norcia, 1994) for each harmonic: for $1 \mathrm{~F}$ (6 Hz), $360 \mathrm{deg}$ corresponds to $167 \mathrm{~ms}$; for $2 \mathrm{~F}$ (12 Hz), 360 deg corresponds to $83.3 \mathrm{~m}$. The apparent latencies derived from the mean $\mathrm{f}_{\min }$ values were then shifted to start at $0 \mathrm{~ms}$ for the $4 \%$ contrast condition. The latency changes from the $4 \%$ contrast condition are thus readily seen. This defines the ordinate. Relative apparent latencies for the $1 \mathrm{~F}$ and $2 \mathrm{~F}$ response components exhibit different contrast dependences.

Phase evidence that the $2 \mathrm{~F}$ components in the VRN and motion control protocols derive from the same cellular mechanisms. Norcia et al. (1999) noted that the high-SNR phase response for a given response component often displays remarkable consistency across conditions that are expected to activate the same cortical visual mechanisms. Such phase reproducibility can even occur between sessions or even between subjects (Chen et al., 2005; Hou et al., 2007). Examples from the present study (i.e., from one individual's sVEP records) are illustrated in Figure 2 (8\% contrast) and Figure 3 (32\% contrast). At each contrast, the response profiles for the two $2 \mathrm{~F}$ phases (motion and VRN protocols) are nearly identical over the entire range of displacement amplitudes.

The group $2 \mathrm{~F}$ phase differences $v s$ contrast in Figure 10A show that the mean differences for high contrasts that are comparable to the parameters used in Norcia et al. (1999) are $25 \mathrm{deg}$ (e.g., at 64\% contrast), corresponding to a latency difference of $5.8 \mathrm{~ms}$ (Figure 10B) between the high-SNR responses for the two protocols.

Similarities in phase responses suggest, but do not prove, that the responses derive from the same neural mechanism, whereas differences in phase responses are stronger evidence that different mechanisms are recruited. The $2 \mathrm{~F}$ phase responses in Figures 2 and 3 and the similarity of the $2 \mathrm{~F}$ phases across stimulus protocols illustrated in Figure 10 are consistent with the $2 \mathrm{~F}$ components being generated by the same mechanism in the two experimental protocols.

Contrast dependence of the effect of gaps on peak amplitude. Gaps increase sVEP $1 \mathrm{~F}$ thresholds (Figure 5 above; Norcia et al., 1999; Hou et al., 2007) and decrease 1F peak amplitudes (Norcia et al., 1999; Hou et al., 2007). These prior measures used highcontrast stimuli. Figure 11 shows the mean $( \pm \mathrm{SE})$ peak amplitudes for the 1F (left panel) and 2F (right panel) response components for four contrasts measured using gaps of 0,2 , or 5 arcmin.

The data presented in the left panel of Figure 11 show that the $1 \mathrm{~F}$ peak amplitudes were extraordinarily sensitive to the spacing of the Vernier elements, especially at high contrast. The $64 \%$ contrast data closely approximated the form of the data of Norcia et al. (1999) (80\% contrast; solid black circles, dotted black lines). The gap effect became less pronounced at lower contrasts.

The right panel in Figure 11 shows comparable peak amplitude data for the $2 \mathrm{~F}$ response component. In contrast to the Vernier (1F) component, we found, similar to Norcia et al. (1999), that the peak amplitude of the motion component (2F) had very little dependence on gap for high-contrast stimuli. Additionally, our data showed that this was also true at lower contrasts, down to $4 \%$.

\section{Discussion}

To our knowledge the present study is the first to examine human cortical Vernier responses using steady-state VEP methods across a range of stimulus contrasts. The threshold measures (Figures $4 \& 5$ ) and suprathreshold measures of peak amplitude (Figure 6), sVEP slope (Figure 7) and gain (Figure 8), and minimum apparent latency (Figure 9) showed that the $1 \mathrm{~F}$ and $2 \mathrm{~F}$ response components had different dependence on contrast. These results support the notion that the $1 \mathrm{~F}$ and $2 \mathrm{~F}$ components derive from non-identical sets of cortical neurons that respond to Vernier offsets and local relative motion, respectively. The $2 \mathrm{~F}$ thresholds are independent of contrast at medium to low contrasts (Figure 4), whereas the $1 \mathrm{~F}$ thresholds steadily decrease with contrast from $4 \%$ to $80 \%$, with a slope of -0.5 on log-log axes (Figure 4 ).

\section{Identification of $1 F$ and $2 F$ response components with Vernier and motion mechanisms}

Overall, the present results strongly support the notion that the $1 \mathrm{~F}$ response components reflect the Vernier-related signals in cortex that are utilized in the psychophysical detection of Vernier offsets at a hyperacuity level (<1 min; Norcia et al., 1999; Chen et al., 2005; Hou et al., 2007).

Effect of gaps on $1 F$ and $2 F$ thresholds and suprathreshold responses. The present study manipulated gaps between stimulus elements and 

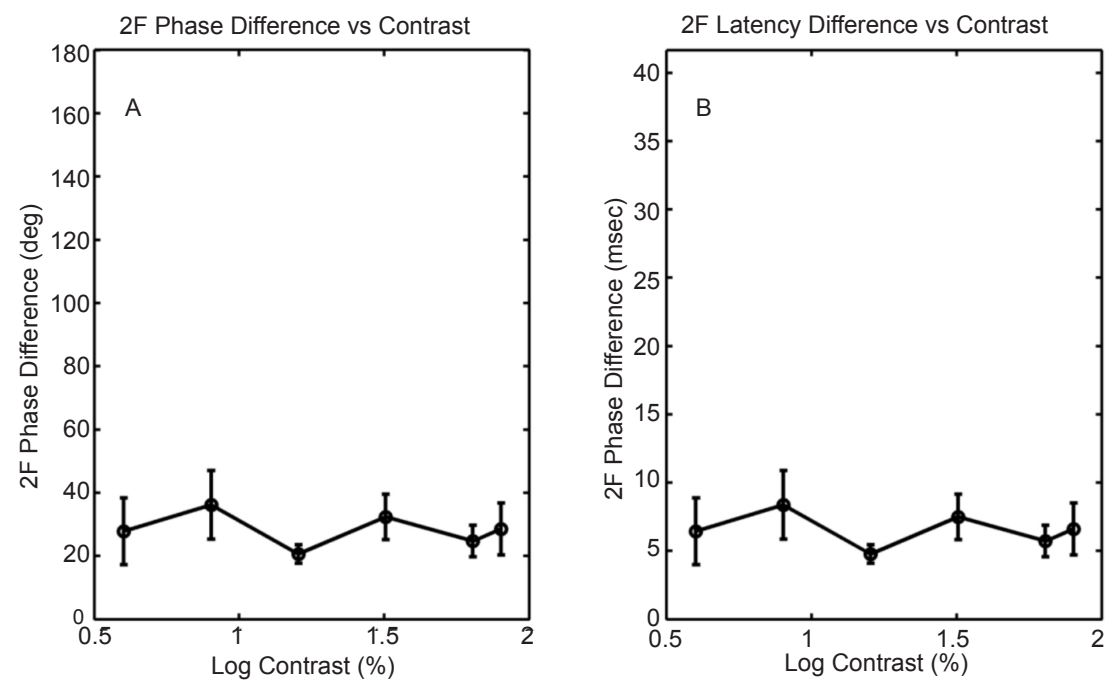

Figure 10. Differences between the VRN and motion control $2 \mathrm{~F}$ phase responses $v s$ stimulus contrast. (A) Mean (unsigned) differences in high-SNR phase (in deg; panel A) and apparent latency (in ms; panel B) for the 2F components. The vertical axis covers a range of $1 / 2$ stimulus cycle ( $\pi$ radians). Notice that the difference between the minimum phases for the two $2 \mathrm{~F}$ components correspond to a mean (unsigned) apparent latency difference of $\sim 6-7 \mathrm{~ms}$, and this difference is independent of stimulus contrast.
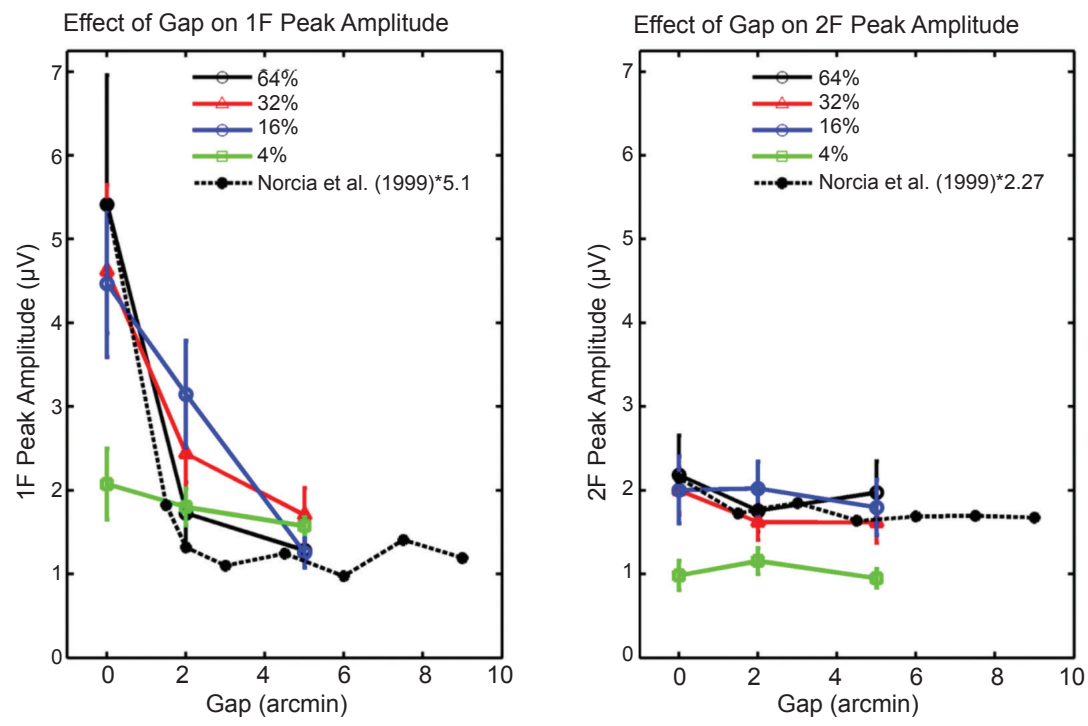

Figure 11. Peak sVEP amplitudes as a function of gap and contrast for $1 \mathrm{~F}$ (left) and $2 \mathrm{~F}$ response components. The peak amplitude of the 1F (Vernier) component decreased with gap size for all contrasts. For the three higher contrasts, the 1F peak amplitude substantially decreased when a gap between Vernier elements was introduced. The $2 \mathrm{~F}$ components derived from the same stimulus and same data record for each subject showed almost no effect of gap for each of the four contrasts tested. The form of our $64 \%$ contrast results closely approximates those of Norcia et al. (1999; filled black circles, dotted black lines). Data from Norcia et al. (1999) were shown as relative amplitude in which all amplitudes were scaled relative to the amplitude for 0 gap (relative amplitude $=1$ ). Thus, data from their figure were multiplied by 5.1 to bring their 0 -gap data into coincidence with our 0 -gap data at $64 \%$ contrast. Data in this figure are from the same subjects whose threshold $v s$ gap results are shown in Figure 5.

confirmed evidence that the $1 \mathrm{~F}$ and $2 \mathrm{~F}$ response components elicited in the steady-state sVEP VRN protocol are dominated by signals that emanate from distinct cortical cells. The $1 \mathrm{~F}$ component reflects the activity of cells that are able to discriminate between the two states of the stimulus (i.e., the Vernier offset [the misalignment event] and the return to alignment; Norcia et al., 1999). Because these two stimulus phases generate different response amplitudes (Zemon \&
Ratliff, 1982; Levi et al., 1983), they generate a strong $1 \mathrm{~F}$ response component. The different responses to the two stimulus states show that the cells are sensitive to the relative spatial phase of the moving and static bar elements.

To discriminate between the two stimulus states, the Vernier-sensitive cells must compare signals across the boundaries of the static and moving bar elements (Norcia et al., 1999). Consistent with this conceptualization, both 
the $1 \mathrm{~F}$ thresholds (Figure 5, left) and $1 \mathrm{~F}$ peak amplitudes (Figure 11, left) are affected by the introduction of gaps between the static and moving bar elements for all of the contrasts tested (i.e., thresholds increase and peak amplitudes decrease). The effect of gaps on sVEP $1 F$ thresholds are qualitatively similar to the effect of gaps on psychophysical (high-contrast) Vernier thresholds (Westheimer \& McKee, 1977; Levi \& Klein, 1982a; Whitaker, 1993; Hou et al., 2007; Sun, Cooper \& Lee, 2012).

The 2F peak amplitudes (Figure 11, right) were unaffected by the gaps for any contrast tested. ${ }^{6}$ The $2 \mathrm{~F}$ thresholds were also unaffected by gaps when contrast was $>4 \%$ (Figure 5). These results are generally consistent with the notion that the $2 \mathrm{~F}$ response components are generated by mechanisms that cannot discriminate between the two states of the VRN stimulus and that do not derive from lateral interactions across the stimulus boundaries.

The one exception was the contrast-dependence of the $2 \mathrm{~F}$ thresholds obtained at $4 \%$ contrast (Figure 5 , right). The reason for this result is unknown. The presence of lateral interactions (implied by the dependence on gap) is counterintuitive because gaps did not affect the thresholds we obtained at $16 \%, 32 \%$, and $64 \%$ contrast. Hou et al. (2007) also found that gaps for high-contrast stimuli did not affect the extrapolated thresholds for the $2 \mathrm{~F}$ component. The $4 \%$-contrast data in Figure 5 (right) are also puzzling because the corresponding peak amplitude data did not change with gap (Figure 11, right). This conundrum will need to be examined in future research.

$1 \mathrm{~F}$ and $2 \mathrm{~F}$ thresholds segregate when contrast is varied. Prior studies used high-contrast stimuli and found that the $1 \mathrm{~F}$ and $2 \mathrm{~F}$ thresholds were similar, suggesting that the Vernier and motion mechanisms had equal sensitivity (Norcia et al., 1999; Chen et al., 2005; Hou et al., 2007). Because the frequency spectrum of asymmetric responses to the periodic Vernier offsets and realignments will contain even harmonics $(2 \mathrm{~F}, 4 \mathrm{~F}$, etc.) in addition to odd-harmonic components, the $2 \mathrm{~F}$ thresholds measured at high contrast may derive from a sum of signals generated by both Vernier-sensitive and motion mechanisms mechanisms in some (as yet unspecified) proportion.

Our data confirmed that $1 \mathrm{~F}$ and $2 \mathrm{~F}$ thresholds are similar at high contrasts. However, when lower contrasts

6. These results are consistent with Norcia et al. (1999) but not with Hou et al. (2007). They found that for high-contrast stimuli, the $2 \mathrm{~F}$ peak amplitudes systematically decreased with gap for gaps between 1' and 4'. This finding differs from the present results (Figure 11) and from prior data from the Norcia laboratory (Norcia et al., 1999). The reason for this discrepancy across studies that used nearly identical stimulus conditions is unknown. The Norcia et al. (1999) and Hou et al. (2007) studies both used lower temporal frequency stimuli ( 3 and $3.76 \mathrm{~Hz}$, respectively) than in the present study $(6 \mathrm{~Hz})$, but the amplitude $v s$. gap results differed in these two studies. are examined, the $1 \mathrm{~F}$ and $2 \mathrm{~F}$ thresholds diverge, consistent with their having different sources (Figure 4).

Suprathreshold response features imply that $1 \mathrm{~F}$ and $2 \mathrm{~F}$ components derive from different neuronal sources with different displacement-gains. At least three suprathreshold response features were strikingly different in the $1 \mathrm{~F}$ and $2 \mathrm{~F}$ components. The $1 \mathrm{~F}$ sVEP peak amplitudes (Figure 6 \& 11), slopes (Figure 7), and relative apparent latencies (Figure 9) all had different contrast dependence than their $2 \mathrm{~F}$ counterparts.

The lack of contrast dependence for the $2 \mathrm{~F}$ slopes is also noteworthy because the extrapolated thresholds over the high- contrast range $(\geq 16 \%)$ decrease with contrast (Figure 4), implying that changing contrast in this range causes parallel shifts of the sVEP data along the vernier-displacement axis. The steeper slopes for the $1 \mathrm{~F}$ components are consistent with the underlying mechanisms having higher gain for the displacement stimulus.

The steeper decline in the $1 \mathrm{~F}$ minimum phases (converted to apparent latencies; Figure 9) shows that the response speed of the mechanisms underlying the $1 \mathrm{~F}$ components increased considerably faster with contrast than the response speed of the $2 \mathrm{~F}$ mechanisms.

\section{The sweep visual evoked potential Vernier threshold data are consistent with the magnocellular hypothesis of Lee and colleagues}

Lee et al. proposed that retinal magnocellular cells but not parvocellular ganglion cells have the requisite properties and sensitivity to absolute stimulus displacements to provide central (cortical) mechanisms with signals that are able to support positional discrimination at the hyperacuity level (e.g., Lee et al., 1993, 1995; Wachtler et al., 1996; Rüttiger \& Lee, 2000; Rüttiger et al., 2002; Sun et al., 2003, 2004; Lee et al., 2005). They noted that $M$ cells' positional thresholds linearly decrease with contrast (on log-log axes), with a slope similar to (or slightly higher than) psychophysical thresholds, depending to some extent on the spatial and temporal frequency of the stimuli (Sun et al., 2003, 2004). P cells, in contrast, have a distinctly different contrast dependence and are much less sensitive to low contrasts. Their responses are almost independent of contrast up to $20-50 \%$ for a range of temporal and spatial frequencies (Sun et al., 2004). Additionally, M SNRs in tests of their spatial precision are substantially higher than SNRs from P cells (Rüttiger et al., 2002; Sun et al., 2004; Lee et al., 2005). M cells' velocity dependence but not P cells' velocity dependence has the same form as human psychophysical Vernier thresholds as a function of stimulus velocity (Rüttiger et al., 2002; Sun et al., 2004; Lee et al., 2005). The M cells' positional thresholds as a function of temporal frequency also have a form more similar to psychophysical thresholds than $\mathrm{P}$ cells' positional thresholds (Sun et al. 2004).

We found that cortical (sVEP) Vernier (1F) thresholds decreased linearly with contrast, with a slope of approximately -0.5 on log-threshold vs log-contrast 
axes (Figure 4). The results paralleled analogous psychophysical data obtained by Sun et al. (2003) and data from several other psychophysical studies of Vernier thresholds (e.g., Watt \& Morgan, 1983; Krauskopf \& Farrell, 1991; Wehrhahn \& Westheimer, 1990; Sun et al., 2004).

Thus, the form of our sVEP Vernier (1F) thresholds as a function of grating contrast, presumably measured at a locus intermediate between retinal ganglion cell responses and the loci that underlie psychophysical detection paralleled both human psychophysical performance and $\mathrm{M}$ cell responses. Therefore, they qualitatively support the magnocellular hypothesis of Lee et al.

Notably, the slope of -0.5 is consistent with the notion that VRN detection is a form of contrast detection and is limited by the same source of noise (Morgan \& Aiba, 1985a; Morgan, 1986; McKee, 1991). The 2F thresholds in Figure 4, which we associate with motion-specific responses, had a different contrast dependence and did not parallel the Vernier psychophysics, especially at low contrasts. A new finding is that the $2 \mathrm{~F}$ thresholds at medium to low contrasts are lower than the $1 \mathrm{~F}$ thresholds.

\section{Clinical considerations}

The measurement of Vernier acuity can provide valuable information for evaluating the effects of and mechanisms underlying a host of visual diseases and vision-threatening conditions. Vernier acuity has been used to evaluate vision in patients with glaucoma (McKendrick, Johnson, Anderson \& Fortune, 2002), patients with schizophrenia (Kéri, Kelemen, Benedek \& Janka, 2004), patients with Down's syndrome (Little, Woodhouse, Lauritzen \& Saunders, 2009), patients with cortical visual impairment (Skoczenski \& Good, 2004; Watson, Orel-Bixler \& Haegerstrom-Portnoy, 2009), and patients and unaffected carriers of the FMRI gene associated with mental retardation in fragile- $X$ syndrome (Keri \& Bendek, 2009, 2011, 2012).

The sVEP measures of Vernier acuity can also be a valuable and objective tool to evaluate vision in populations in whom reliable optotype acuity cannot be readily attained (e.g., preverbal infants and toddlers and multiply-handicapped patients; Mirabella et al., 2006; Hou et al., 2011). A notable example of the application of Vernier measures in disease is the characterization of amblyopia. The evaluation of Vernier and other hyperacuity tasks has proven to be valuable in the overall assessment of amblyopia (Levi \& Klein, 1982a,b, 1985; Bradley \& Freeman, 1985; Levi, Klein \& Yap, 1987; Birch \& Swanson, 2000; McKee, Levi \& Movshon, 2003; Chen et al., 2005; Hou et al., 2007). Several psychophysical studies have shown that Vernier acuity is more strongly affected in amblyopia than grating acuity. Additionally, Vernier acuity and deficits therein correlate better with optotype acuity than grating acuity (e.g., Bradley \& Freeman, 1985; Birch \& Swanson, 2000; McKee et al., 2003).

Vernier acuity assessment is thus a natural additional tool for detecting and quantifying amblyopia.
Measuring Vernier acuity along with grating acuity and contrast sensitivity can provide a more useful profile of visual function because assessments of grating acuity alone can underestimate the functional loss of (optotype) acuity (e.g., Levi, Klein \& Wang, 1994a,b; McKee et al., 2003).

In addition to the threshold measures cited above, Hou et al. (2007) found that strabismic amblyopes's VEP Vernier suprathreshold (peak) amplitudes were lower than anisometropic amblyopes' in both the amblyopic and fellow eyes, although on average the two groups had equal Vernier (1F) thresholds and visual acuities in both sVEP and psychophysical measures. These results highlight the fact that suprathreshold features of VEP responses can provide additional information independent of threshold and may be able to reveal effects of diseases or experimental manipulations when psychophysical/perceptual measures fail to do so. Suprathreshold amplitude and phase information may thus aid in characterizing different diseases.

Vernier measures may help clarify possible specific neuronal targets in disease mechanisms. Visual evoked potential Vernier measures may help characterize neuronal specificity (or lack thereof) in various disease conditions. For example, alterations in Vernier and contrast sensitivity have been hypothesized to implicate preferential magnocellular involvement (e.g., Keri \& Benedek, 2009). Vernier acuity is reduced in patients with fragile-X syndrome (e.g., Keri \& Benedek, 2009, 2011, 2012), which appears to preferentially affect magnocellular pathways (Kogan et al., 2004).

Although the link between Vernier performance and magnocellular deficits is not without controversy (e.g., Skottun \& Skoyles, 2004, 2007a,b,c,d, 2010a,b), the present findings should help clarify this issue as research on this topic continues. Thus, measurement of Vernier sVEP responses provides a sensitive tool to expand the repertoire of visual measures that we can apply to evaluate the impact of visual disease processes and the effectiveness of clinical interventions.

\section{Acknowledgements}

We are grateful to Profs. Dingcai Cao, Vance Zemon, and Marcelo F. da Costa for assistance with the statistical analyses. This research was supported by funds from the Brazilian agencies FAPESP (no. 02007/52321-4, 02/12733-8, and 2008/58731-2) and CNPq (no. 302527/2008-7).

\section{References}

Alexander, B. F. \& Ng, K. C. (1991). Elimination of systematic error in subpixel accuracy centroid estimation. Optical Engineering, 30, 1320-1331.

Birch, E. E. \& Swanson, W. H. (2000). Hyperacuity deficits in anisometropic and strabismic amblyopes with known ages of onset. Vision Research, 40, 1035-1040.

Birch, E. E., Fawcett, S. \& Stager, D. (2000). Co-development of VEP motion response and binocular vision in normal infants and infantile esotropes. Investigative Ophthalmology and Visual Science, 41, 1719-1723. 
Bradley, A. \& Freeman, R. D. (1985) Is reduced vernier acuity in amblyopia due to position, contrast or fixation deficits? Vision Research, 25, 55-66.

Bradley, A. \& Skottun, B. C. (1987). Effects of contrast and spatial frequency on vernier acuity. Vision Research, 27, 1817-1824.

Campbell, F. W. \& Maffei, L. (1970). Electrophysiological evidence for the existence of orientation and size detectors in the human visual system. Journal of Physiology, 207, 635-652.

Carney, T. \& Klein, S. A. (1997). Resolution acuity is better than vernier acuity. Vision Research, 37, 525-539.

Chen, S. I., Norcia, A. M., Pettet, M. W. \& Chandna, A. (2005). Measurement of position acuity in strabismus and amblyopia: Specificity of the vernier VEP paradigm. Investigative Ophthalmology and Visual Science, 46, 4563-4570.

Good, W. V. \& Hou, C. (2004). Normal vernier acuity in infants with delayed visual maturation. American Journal of Ophthalmology, $138,140-142$.

Hamer, R. D. \& Norcia, A. M. (1994). The development of motion sensitivity during the first year of life. Vision Research, 34, 23872402 .

Hering, E. (1899). Uber die Grenzen der Sehscharfe. Ber. math-phys. Cl. d. Konigl. Sachs. Gesell. Wiss. Leipzig; Naturwiss. Teil 16-24.

Hou, C., Good, W. V. \& Norcia, A. M. (2007). Validation study of VEP vernier acuity in normal-vision and amblyiopic adults. Investigative Ophthalmology and Visual Science, 49, 4070-4078.

Hou, C., Norcia, A. M., Madan, A., Tith, S., Agarwal, R. \& Good, W. V. (2011). Visual cortical function in very low birth weight infants without retinal or cerebral pathology. Investigative Ophthalmology \& Visual Science, 52, 9091-9098.

Hu, Q., Klein, S. A. \& Carney, T. (1993). Can sinusoidal vernier acuity be predicted by contrast discrimination? Vision Research, 33, 12411258

Jampolsky, A., Norcia, A. M. \& Hamer, R. D. (1994). Preoperative alternate occlusion decreases motion processing abnormalities in infantile esotropia. Journal of Pediatric Ophthalmology and Strabismus, 31, 6-17.

Kaplan, E. \& Shapley, R. M. (1982). X and Y cells in the lateral geniculate nucleus of macaque monkeys. Journal of Physiology, 330, 125-143.

Kaplan, E. \& Shapley, R. M. (1986). The primate retina contains two types of ganglion cells, with high and low contrast sensitivity. Proceedings of the National Academy of Sciences of the United States of America, 83, 2755-2757.

Keri, S. \& Benedek, G. (2009). Visual pathway deficit in female fragile X premutation carriers. A potential endophenotype. Brain and Cognition, 69, 291-295.

Kéri, S. \& Benedek, G. (2011). Fragile X protein expression is linked to visual functions in healthy male volunteers. Neuroscience, 192, 345-350.

Kéri, S. \& Benedek, G. (2012). Why is vision impaired in fragile $\mathrm{X}$ premutation carriers? The role of fragile $\mathrm{X}$ mental retardation protein and potential FMR1 mRNA toxicity. Neuroscience, 206, 183-189.

Kéri, S., Kelemen, O., Benedek, G. \& Janka, Z. (2004). Vernier threshold in patients with schizophrenia and in their unaffected siblings. Neuropsychology, 18, 537-542.

Klein, S. A. \& Levi, D. M. (1985). Hyperacuity thresholds of $1 \mathrm{sec}$ Theoretical predictions and empirical validation. Journal of the Optical Society of America A, 2, 1170-1190.

Kogan, C. S., Boutet, I., Cornish, K., Zangenehpour, S., Mullen, K. T., Holden, J. J., ... Chaudhuri, A. (2004). Differential impact of the FMR1 gene on visual processing in fragile $\mathrm{X}$ syndrome. Brain, 127, 591-601.

Krauskopf, J. \& Farrell, B. (1991). Vernier acuity: Effects of chromatic content, blur and contrast. Vision Research, 31, 735-749.

Krotkov, E. P. (1986). Visual hyperacuity: Representation and computation of high precision position information. Computer Vision, Graphics, and Image Processing, 33, 99-115.

Lee, B. B., Rüttiger, L. \& Sun, H. (2005). Comparison of ganglion cell signals and psychophysical localization of moving targets can help define central motion mechanisms. Perception, 34, 975-981.

Lee, B. B., Wehrhahn, C., Westheimer, G. \& Kremers, J. (1993). Macaque ganglion cell responses to stimuli that elicit hyperacuity in man: Detection of small displacements. Journal of Neuroscience, 13, 1001-1009.

Lee, B. B., Wehrhahn, C., Westheimer, G. \& Kremers, J. (1995). The spatial precision of macaque ganglion cell responses in relation to vernier acuity of human observers. Vision Research, 35, 2743-2758.

Levi, D. M. \& Klein S. (1982a). Differences in vernier discrimination for grating between strabismic and anisometropic amblyopes. Investigative Ophthalmology and Visual Science, 23, 398-407.

Levi, D. M. \& Klein, S. (1982b). Hyperacuity and amblyopia. Nature, 298, 268-270.

Levi, D. M. \& Klein, S. A. (1985). Vernier acuity, crowding and amblyopia. Vision Research, 25, 979-991.

Levi, D. M., Klein, S. A. \& Carney, T. (2000). Unmasking the mechanisms for Vernier acuity: Evidence for a template model for Vernier acuity. Vision Research, 40, 951-972.

Levi, D. M., Klein, S. A. \& Wang, H. (1994a). Amblyopic and peripheral vernier acuity: A test-pedestal approach. Vision Research, 34, 3265-3292.

Levi, D. M., Klein, S. A. \& Wang, H. (1994a). Discrimination of position and contrast in amblyopic and peripheral vision. Vision Research, 34, 3293-3313.

Levi, D. M., Klein, S. A. \& Yap, Y. L. (1987). Positional uncertainty in peripheral and amblyopic vision. Vision Research, 27, 581-597.

Levi, D. M., Manny, R. E., Klein, S. A. \& Steinman, S. B. (1983). Eletrophysiological correlates of hyperacuity in the human visual cortex. Nature, 306, 468-470.

Little, J. A., Woodhouse, J. M., Lauritzen, J. S. \& Saunders, K. J. (2009). Vernier acuity in Down syndrome. Investigative Ophthalmology and Visual Science, 50, 567-572.

Manny, R. E. (1988). The visually evoked potential in response to vernier offsets in infants. Human Neurobiology, 6, 273-279.

McKee, S. P. (1991). The physical constraints on visual hyperacuity. In J. J. Kulikowski, V. Walsh \& I. J. Murray (Eds.), Limits of vision (series title: Vision and visual dysfunction, vol. 5) (pp. 221-233). Boca Raton, FL: CRC Press.

McKee, S. P., Levi, D. M. (1987). Dichoptic hyperacuity: The precision of nonius alignment. Journal of the Optical Society of America A, 4,1104-1108.

McKee, S. P., Levi, D. M. \& Movshon, J. A. (2003) The pattern of visual deficits in amblyopia. Journal of Vision, 3, 380-405.

McKendrick, A. M., Johnson, C. A., Anderson, A. J. \& Fortune, B. (2002). Elevated vernier acuity thresholds in glaucoma. Investigative Ophthalmology and Visual Science, 43, 1393-1399.

Morgan, M. J. (1986). Positional acuity without monocular cues. Perception, 15, 157-162.

Medeiros, A., Farias de Medeiros, C. \& Monteiro, F. N., Jr. (2004). Pedro nunes e o problema histórico da compreensão da medição das frações. Ciência \& Educação, 10, 559-570.

Mirabella, G., Kjaer, P. K., Norcia, A. M., Good, W. V. \& Madan, A. (2006). Visual development in very low birth weight infants. Pediatric Research, 60, 435-439.

Morgan, M. J. \& Aiba, T. S. (1985a). Positional acuity with chromatic stimuli. Vision Research, 25, 689-695.

Morgan, M. J. \& Aiba, T. S. (1985b). Vernier acuity predicted from changes in the light distribution of the retinal image. Spatial Vision, $1,151-161$

Morgan, M. J. \& Watt, R. J. (1984). Spatial frequency interference effects and interpolation in vernier acuity. Vision Research, 24, 1911-1919.

Morgan, M. J., Watt, R. J. \& McKee, S. P. (1983). Exposure duration affects the sensitivity of vernier acuity to target motion. Vision Research, 23, 541-546.

Nelson, J. I., Seiple W. H., Kupersmith, M. J. \& Carr, R. E. (1984). A rapid evoked potential index of cortical adaptation. Electroencephalography and Clinical Neurophysiology, 59, 454464.

Norcia, A. M. \& Tyler, C. W. (1985). Spatial frequency sweep VEP: Visual acuity during the first year of life. Vision Research, 25, 13991408 .

Norcia, A. M., Garcia, H., Humphry, R., Holmes, A., Hamer, R. D. \& Orel-Bixler, D. (1991). Anomalous motion VEPs in infants and in infantile esotropia. Investigative Ophthalmology and Visual Science, 32, 436-439. 
Norcia, A. M., Hamer, R. D., Jampolsky, A. \& Orel-Bixler, D. (1995). Plasticity of human motion processing mechanisms following surgery for infantile esotropia. Vision Research, 35, 3279-3296.

Norcia, A. M., Tyler, C. W., Hamer, R. D. \& Wesemann, W. (1989). Measurement of spatial contrast sensitivity with the swept contrast VEP. Vision Research, 29, 627-637.

Norcia, A. M., Wesemann, W. \& Manny, R. E. (1999). Eletrophysiological correlates of vernier and relative motion mechanisms in human visual cortex. Visual Neuroscience, 16, 1123-1131.

Odom, J. V., Bach, M., Barber, C., Brigell, M., Marmor, M. F., Tormene, A. P., ... Vaegan (2004). Visual evoked potentials standard (2004). Documenta Ophthalmologica, 108, 115-123.

Purpura, K., Kaplan, E. \& Shapley, R. M. (1988). Background light and contrast gain of primate $\mathrm{P}$ and $\mathrm{M}$ retinal ganglion cells. Proceedings of National Academy of Sciences of the United States of America, 85, 4534-4537.

Regan, D. (1973). Rapid objective refraction using evoked brain potentials. Investigative Ophthalmology and Visual Science, 12, 669-679.

Regan, D. (1989). Human brain electrophysiology: evoked potentials and evoked magnetic fields in science and medicine. New York: Elsevier.

Rüttiger, L. \& Lee, B. B. (2000). Chromatic and luminance contributions to a hyperacuity task. Vision Research, 40, 817-832.

Rüttiger, L., Lee, B. \& Sun, H. (2002). Transient cells can be neurometrically sustained: The positional accuracy of retinal signals to moving targets. Journal of Vision, 2, 232-242.

Shea, S. J., Chandna, A. \& Norcia, A. M. (1999). Oscillatory motion but not pattern reversal elicits monocular motion VEP biases in infantile esotropia. Vision Research, 39, 1803-1811.

Skoczenski, A. M. \& Good, W. V. (2004). Vernier acuity is selectively affected in infants and children with cortical visual impairment. Developmental Medicine and Child Neurology, 46, 526-532.

Skoczenski, A. M. \& Norcia, A. M. (1999). Development of VEP Vernier acuity and grating acuity in human infants. Investigative Ophthalmology and Visual Science, 40, 2411-2417.

Skoczenski, A. M. \& Norcia, A. M. (2002). Late maturation of visual hyperacuity. Psychological Science, 13, 537-541.

Skottun, B. C. \& Skoyles, J. R. (2004). Some remarks on the use of motion VEPs to assess magnocellular sensitivity. Clinical Neurophysiology, 115, 2834-2836.

Skottun, B. C. \& Skoyles, J. R. (2007a). Dyslexia, direction selectivity and magnocellular sensitivity. Vision Research, 47, 1974-1975.

Skottun, B. C. \& Skoyles, J. R. (2007b). Contrast sensitivity and magnocellular functioning in schizophrenia. Vision Research, 47, 2923-2933.

Skottun, B. C. \& Skoyles, J. R. (2007c). Some remarks on the use of visually evoked potentials to measure magnocellular activity. Clinical Neurophysiology, 118, 1903-1905.

Skottun, B. C. \& Skoyles, J. R. (2007d). A few remarks on assessing magnocellular sensitivity in schizophrenic patients. Brain, 130, e83.

Skottun, B. C. \& Skoyles, J. R. (2010a). On interpreting responses to low contrast stimuli in terms of magnocellular activity: A few remarks. Vision Research, 50, 989-990.

Skottun, B. C. \& Skoyles, J. R. (2010b). On using Vernier acuity to assess magnocellular sensitivity. Brain and Cognition, 72, 165-166.

Steinman, S. B., Levi, D. M., Klein, S. A. \& Manny, R. E. (1985). Selectivity of the evoked potential for vernier offset. Vision Research, 25, 951-961.

Sun, H., Cooper, B. \& Lee, B. B. (2012). Luminance and chromatic contributions to a hyperacuity task: Isolation by contrast polarity and target separation. Vision Research, 56, 28-37.

Sun, H., Lee, B. B. \& Baraas, R. C. (2008). Systematic misestimation in a vernier task arising from contrast mismatch. Visual Neuroscience, $25,365-370$.

Sun, H., Lee, B. \& Rüttiger, L. (2003). Coding of position of achromatic and chromatic edges by retinal ganglion cells. In: J. D.
Mollon, I. Pokorny \& K. Knoblauch (Eds.), Normal and defective colour vision (pp. 79-87). Oxford: Oxford University Press.

Sun, H., Rüttiger, L. \& Lee, B. B. (2004). The spatiotemporal precision of ganglion cell signals: A comparison of physiological and psychophysical performance with moving gratings. Vision Research, 44, 19-33.

Tang, Y. \& Norcia, A. M. (1993). Improved processing of the steadystate evoked potential. Electroencephalography and Clinical Neurophysiology, 88, 323-334.

Tang, Y. \& Norcia, A. M. (1995). An adaptive filter for steadystate evoked responses. Electroencephalography and Clinical Neurophysiology, 96, 268-277.

Tyler, C. W., Apkarian, P., Levi, D. M. \& Nakayama, K. (1979). Rapid assessment of visual function: An electronic sweep technique for the pattern visual evoked potential. Investigative Ophthalmology and Visual Science, 18, 703-713.

Victor, J. D. \& Conte, M. M. (2000). Two-frequency analysis of interactions elicited by Vernier stimuli. Visual Neuroscience, 17, 959-973.

Victor, J. D. \& Mast J. (1991). A new statistic for steady-state evoked potentials. Electroencephalography \& Clinical Neurophysiology, 78, 378-388.

Wachtler, T., Wehrhahn, C. \& Lee, B. B. (1996). A simple model of human foveal ganglion cell responses to hyperacuity stimuli. Journal of Computational Neuroscience, 3, 73-82.

Watson, T., Orel-Bixler, D. \& Haegerstrom-Portnoy, G. (2009). VEP vernier, VEP grating, and behavioral grating acuity in patients with cortical visual impairment. Optometry and Vision Science, 86, 774780 .

Watt, R. J. \& Morgan, M. J. (1983). Mechanisms responsible for the assessment of visual location: Theory and evidence. Vision Research, 23, 97-109.

Waugh, S. J. \& Levi, D. M. (1993a). Visibility and vernier acuity for separated targets. Vision Research, 33, 539-552.

Waugh, S. J. \& Levi, D. M. (1993b). Visibility, luminance and vernier acuity. Vision Research, 33, 527-538.

Waugh, S. J. \& Levi, D. M. (1993c). Visibility, timing and vernier acuity. Vision Research, 33, 505-526.

Wehrhahn, C. \& Westheimer, G. (1990). How vernier acuity depends on contrast. Experimental Brain Research, 80, 618-620.

Wesemann,W., Norcia, A. M. \& Manny, R. E. (1996). Messung der Noniussehscharfe und der Bewegungswahrnehmung mit dem Parameter-Sweep-VEP. Klinische Monatsblatter fur Augenheilkunde, 208, 11-17.

Westheimer, G. \& Hauske, G. (1975). Temporal and spatial interference with vernier acuity. Vision Research, 15, 1137-1141

Westheimer, G. \& McKee, S. P. (1977). Spatial configurations for visual hyperacuity. Vision Research, 17, 941-947.

Whitaker, D. (1993). What part of a vernier stimulus determines performance? Vision Research, 33, 27-32.

Wilson, H. R. (1986). Responses of spatial mechanisms can explain hyperacuity. Vision Research, 26, 453-469.

Wilson, H. R. (1991). Model of peripheral and amblyopic hyperacuity. Vision Research, 31, 967-982

Wulfing, E. A. (1892). Uber den kleinsten Gesicbtswinkel. Zeitschrift fur Biologie, 29, 199-202.

Zak, R. \& Berkley, M. A. (1986). Evoked potentials elicited by brief vernier offsets: Estimating vernier thresholds and properties of the neural substrate. Vision Research, 26, 439-451.

Zemon, V. \& Ratliff, F. (1982). Visual evoked potentials: Evidence for lateral interactions. Proceedings of the National Academy of Sciences of the United States of America, 79, 5723-5726.

Zemon, V. \& Ratliff, F. (1984). Intermodulation components of the visual evoked potential: Responses to lateral and superimposed stimuli. Biological Cybernetics, 50, 401-408.

Zemon, V., Victor, J. D. \& Ratliff, F. (1986). Functional subsystems in the visual pathways of humans characterized using evoked potentials. In R. Q. Cracco \& I. Bodis-Wollner (Eds.), Evoked potentials (pp. 203-210). New York: Alan R. Liss. 